\title{
ON PRODUCTS OF HARMONIC POLYNOMIALS
}

\author{
TOMAS SCHONBEK
}

(Communicated by Barbara L. Keyfitz)

\begin{abstract}
We prove that every polynomial in $n$ variables, $n \geq 2$, is a finite sum of terms, each of which is the product of two harmonic polynomials. This strengthens a result obtained by A. G. Ramm in [1].
\end{abstract}

\section{INTRODUCTION}

In [1], A. G. Ramm proves that if $f: \mathbf{R}^{3} \rightarrow \mathbf{C}$ is compactly supported and

$$
\int f(x) P(x) Q(x) d x=0
$$

for all choices of harmonic polynomials $P, Q$, then $f=0$. Using a formula giving the development of a product of two spherical harmonics into a sum of spherical harmonics, Ramm shows that if $f$ satisfies (1) and if $Y$ is a spherical harmonic, then there exists a sequence of positive exponents $\left\{p_{i}\right\}$ (which may depend on $Y$ ) such that $\sum_{i}\left(1 / p_{i}\right)=\infty$ and such that

$$
\int_{0}^{R} r^{p_{i}} \int_{S^{2}} f(r w) Y(w) d w d r=0
$$

for all $i$, where $R>0$ is such that the ball of center 0 with radius $R$ contains the support of $f$ and $S^{2}$ is the unit sphere in $\mathbf{R}^{3}$. By the Müntz-Szasz theorem (cf., for example, [6]), it follows that $w \rightarrow f(r w)$ is orthogonal to all harmonic polynomials for each $r$, hence zero. He then shows how to prove a considerably more general theorem. The general proof turns out to be quite simple. Results such as this one were used very successfully (and ingeniously) by Ramm to settle several open questions in inverse scattering theory (cf., for example, [2-5]). A natural question in this context, which neither the particular proof sketched above nor the more general one answers, is which polynomials can be expressed as a finite sum of terms, each of which is the product of two harmonic polynomials. We prove in this paper that if the number of variables is larger than or equal to 2 , then every polynomial can be so expressed. We

Received by the editors April 14, 1989 and, in revised form, November 3, 1989.

1980 Mathematics Subject Classification (1985 Revision). Primary 35J05, 41A10; Secondary $15 \mathrm{~A} 03$. 
state the theorem and introduce the notation to be used in $\S 2$; the theorem is proved in $\S \S 3$ and 4.

\section{THE MAIN THEOREM}

Let $n$ be a positive integer. For $k=0,1,2, \ldots$, we denote by $\mathscr{P}_{k}(n)$ the space of all polynomials with complex coefficients of the variable $x=$ $\left(x_{1}, \ldots, x_{n}\right) \in \mathbf{R}^{n}$ which are homogeneous of degree $k$. We denote by $\mathscr{H}_{k}(n)$ the subspace of $\mathscr{P}_{k}(n)$ consisting of all $P$ such that $\Delta P=0$, where $\Delta=$ $\sum_{j} \partial^{2} / \partial x_{j}^{2}$ is the Laplacian. We set $\mathscr{P}(n)=\bigcup_{k \geq 0} \mathscr{P}_{k}(n), \mathscr{H}(n)=\bigcup_{k \geq 0} \mathscr{H}_{k}(n)$ and let $\mathscr{H}^{2}(n)$ be the subspace of $\mathscr{P}(n)$ spanned by all polynomials $P Q$ where $P, Q \in \mathscr{H}(n)$. Our theorem can then be stated as follows.

Theorem. If $n \geq 2$, then $\mathscr{H}^{2}(n)=\mathscr{P}(n)$.

\section{THE CASE $n=2$}

The proof of the theorem will be by induction on the dimension $n$. In this section, we prove the theorem for the case $n=2$.

For $k=1,2, \ldots$, let

$$
\begin{aligned}
& P_{k}(x, y)=\sum_{j=0}^{[k / 2]}(-1)^{j}\left(\begin{array}{c}
k \\
2 j
\end{array}\right) x^{k-2 j} y^{2 j} \\
& Q_{k}(x, y)=\frac{1}{k} \sum_{j=0}^{[(k-1) / 2]}(-1)^{j}\left(\begin{array}{c}
k \\
2 j+1
\end{array}\right) x^{k-2 j-1} y^{2 j+1},
\end{aligned}
$$

where [ ] denotes the greatest integer function. It is easy to see that $P_{k}$, $Q_{k} \in \mathscr{H}_{k}(2)$ (and are, in fact, a basis for $\mathscr{H}_{k}(2)$ ). Assume now $k=2 m$ or $k=2 m+1$. Set $R_{j}=P_{j} P_{k-j}, S_{j}=P_{j} Q_{k-j}$ for $j=1, \ldots, m$. We claim that the polynomials

$$
P_{k}, Q_{k}, R_{1}, \ldots, R_{m}, S_{1}, \ldots, S_{m-1}
$$

are linearly independent in $\mathscr{P}_{k}(2)$ if $k=2 m$ is even; in case $k=2 m+1$ is odd, we claim that the polynomials

$$
P_{k}, Q_{k}, R_{1}, \ldots, R_{m}, S_{1}, \ldots, S_{m}
$$

are linearly independent in $\mathscr{P}_{k}(2)$. In either case we have $k+1=\operatorname{dim} \mathscr{P}_{k}(2)$ polynomials; thus the theorem will be proved for the case $n=2$ once these claims are established.

To establish the claims, we will need to evaluate $\Delta^{r} R_{j}, \Delta^{r} S_{j}$ for $1 \leq j \leq r \leq$ $m$. To accomplish this, we begin by proving the following: If $f, g: \mathbf{R}^{2} \rightarrow \mathbf{C}$ are harmonic, then for $r=0,1,2, \ldots$,

$$
\Delta^{r}(f g)=2^{r} \sum_{j=0}^{r}\left(\begin{array}{l}
r \\
j
\end{array}\right) \frac{\partial^{r} f}{\partial x^{j} \partial y^{r-j}} \frac{\partial^{r} g}{\partial x^{j} \partial y^{r-j}} .
$$

The formula is immediate if $r=0$. For $0 \leq j \leq r$, set

$$
F_{r, j}=\frac{\partial^{r} f}{\partial x^{j} \partial y^{r-j}} \frac{\partial^{r} g}{\partial x^{j} \partial y^{r-j}},
$$


so that (3) becomes $\Delta^{r}(f g)=2^{r} \sum_{j=0}^{r}\left(\begin{array}{c}r \\ j\end{array}\right) F_{r, j}$, and assume (3) proved for some $r \geq 0$. Since $\left(\partial^{r} / \partial x^{j} \partial y^{r-j}\right) f,\left(\partial^{r} / \partial x^{j} \partial y^{r-j}\right) g$ are again harmonic, we see $\Delta F_{r, j}=2 F_{r+1, j+1}+2 F_{r+1, j}$ so that, by the induction hypothesis,

$$
\begin{aligned}
\Delta^{r+1}(f g) & =2^{r} \sum_{j=0}^{r}\left(\begin{array}{c}
r \\
j
\end{array}\right) \Delta F_{r, j}=2^{r+1}\left[\sum_{j=0}^{r}\left(\begin{array}{l}
r \\
j
\end{array}\right) F_{r+1, j+1}+\sum_{j=0}^{r}\left(\begin{array}{l}
r \\
j
\end{array}\right) F_{r+1, j}\right] \\
& =2^{r+1} \sum_{j=0}^{r+1}\left(\begin{array}{c}
r+1 \\
j
\end{array}\right) F_{r+1, j},
\end{aligned}
$$

which is (3) with $r$ replaced by $r+1$. By (3), $\Delta^{r}(P Q)=0$ if $P, Q$ are harmonic polynomials and the degree of $P$ (or $Q$ ) is less than $r$. In particular, $\Delta^{r} R_{j}=\Delta^{r} S_{j}=0$ if $r>j$.

Now assume $0 \leq j \leq r \leq k$; then

(4) $\frac{\partial^{r} P_{k}}{\partial x^{j} \partial y^{r-j}}=\sum_{r-j \leq 2 \nu \leq k-j}(-1)^{\nu} \frac{k !}{(k-2 \nu-j) !(2 \nu-r+j) !} x^{k-2 \nu-j} y^{2 \nu-r+j}$.

Specializing to $k=r$, we get

$$
\frac{\partial^{r} P_{r}}{\partial x^{j} \partial y^{r-j}}= \begin{cases}0 & \text { if } r-j \text { is odd, } \\ r !(-1)^{(r-j) / 2} & \text { if } r-j \text { is even. }\end{cases}
$$

By (3) and (5), to evaluate $\Delta^{r}\left(R_{r}\right)=\Delta^{r}\left(P_{r} S_{k-r}\right), \Delta^{r}\left(S_{r}\right)=\Delta^{r}\left(P_{r} Q_{k-r}\right)$, we only need to compute $\left(\partial^{r} / \partial x^{j} \partial y^{r-j}\right)$ applied to $P_{k-r}, Q_{k-r}$ in case $r-j$ is even. Assuming, thus, from now on that $r-j$ is even and replacing $k$ by $k-r$ in (4), we get for $0 \leq j \leq r \leq k-r$,

$$
\frac{\partial^{r} P_{k-r}}{\partial x^{j} \partial y^{r-j}}=(-1)^{(r-j) / 2} p_{k, r}(x, y),
$$

where

$$
p_{k, r}(x, y)=\sum_{\nu=0}^{[(k-2 r) / 2]}(-1)^{\nu} \frac{(k-r) !}{(k-2 r-2 \nu) !(2 \nu) !} x^{k-2 r-2 \nu} y^{2 \nu} .
$$

All that will matter about $p_{k, r}$ is that its coefficients do not depend on $j$ and that the highest appearing power of $x$ is $k-2 r$. A similar computation shows if $0 \leq j \leq r \leq k-r-1$, then

$$
\frac{\partial^{r} Q_{k-r}}{\partial x^{j} \partial y^{r-j}}=(-1)^{(r-j) / 2} q_{k, r}(x, y),
$$

where

$$
q_{k, r}(x, y)=\sum_{\nu=0}^{[(k-2 r-1) / 2]}(-1)^{\nu} \frac{(k-r-1) !}{(k-2 r-2 \nu-1) !(2 \nu+1) !} x^{k-2 r-2 \nu-1} y^{2 \nu+1}
$$


is a polynomial independent of $j$ of degree $k-2 r-1$ in $x$. By (3), (5), (6), if $k \geq 2 r$,

$$
\Delta^{r}\left(R_{r}\right)=([r / 2]+1) r ! p_{k, r}(x, y) ;
$$

by (3), (5), (7), if $k \geq 2 r+1$,

$$
\Delta^{r}\left(S_{r}\right)=([r / 2]+1) r ! q_{k, r}(x, y) .
$$

Since $p_{k, r}, q_{k, r}$ have different degrees as polynomials in $x$, we notice that if $\alpha, \beta$ are complex numbers and $\alpha \Delta^{r}\left(R_{r}\right)+\beta \Delta^{r}\left(S_{r}\right)=0$, then $\alpha=\beta=0$. Let now $S$ be a linear combination of the polynomials in (2e) or $(20)$. Then

$$
S=a P_{k}+b Q_{k}+\sum_{r=1}^{m} c_{r} R_{r}+\sum_{r=1}^{m-1} d_{r} S_{r}
$$

if $k=2 m$ is even;

$$
S=a P_{k}+b Q_{k}+\sum_{r=1}^{m} c_{r} R_{r}+\sum_{r=1}^{m} d_{r} S_{r}
$$

if $k=2 m+1$ is odd. Assuming $S=0$, we have $\Delta^{m} S=0$. Since $\Delta^{m} R_{r}=$ $\Delta^{m} S_{r}=0$ if $m>r$, we are left with $c_{m} \Delta^{m} R_{m}=0$ in the even case and $c_{m} \Delta^{m} R_{m}+d_{m} \Delta^{m} S_{m}=0$ in the odd case. Thus $c_{m}=0$ and (in the odd case) $d_{m}=0$. Applying now $\Delta^{m-1}, \ldots, \Delta$ to $S$, we get successively $c_{r} \Delta^{r} R_{r}+$ $d_{r} \Delta^{r} S_{r}=0$, hence $c_{r}=d_{r}=0$ for $r=m-1, \ldots, 1$. Finally, $a$ and $b$ must be 0 , since $P_{k}, Q_{k}$ are independent. The claim is established, proving the theorem in the case $n=2$.

\section{THE General CASE}

For the induction step, we need the following lemma:

Lemma. Let $k \geq 0$. If $P \in \mathscr{P}_{k}(n)$, then $P=\Delta Q$, for some $Q \in \mathscr{P}_{k+2}(n)$.

Proof. Perhaps the easiest way to prove this result is introducing in $\mathscr{P}_{k}$ the inner product $\langle P, Q\rangle=\sum_{|\alpha|=k} \alpha ! a_{\alpha} b_{\alpha}$ if $P(x)=\sum_{|\alpha|=k} a_{\alpha} x^{\alpha}, Q(x)=\sum_{|\alpha|=k} b_{\alpha} x^{\alpha}$ (where $\alpha !=\alpha_{1} ! \cdots \alpha_{n} !,|\alpha|=\alpha_{1}+\cdots+\alpha_{n}$ if $\alpha=\left(\alpha_{1}, \ldots, \alpha_{n}\right)$ is a multi-index). An equivalent way of defining this inner product is by $\langle P, Q\rangle=P(D) \bar{Q}$, where $P(D)$ is the operator $\sum_{|\alpha|=k} a_{\alpha} D^{\alpha}$ if $P=\sum_{|\alpha|=k} a_{\alpha} x^{\alpha}$ (cf, [7, p. 69]). In this formulation, once sees at once that this inner product satisfies the following: Let $P \in \mathscr{P}_{k}(n), R \in \mathscr{P}_{\nu}(n)$, and $Q \in \mathscr{P}_{k+\nu}(n)$; then $\langle R P, Q\rangle=\langle P, \bar{R}(D) Q\rangle$. Assuming now the conclusion of the Lemma to be false, there exists a nonzero $P \in \mathscr{P}_{k}$ such that $\langle P, \Delta Q\rangle=0$ for all $Q \in \mathscr{P}_{k+2}$. However, this implies $\left\langle|x|^{2} P, Q\right\rangle=0$ for all $Q \in \mathscr{P}_{k+2}$, hence $|x|^{2} P=0$; thus also $P=0$. The Lemma is proved.

Assume now the theorem has been proved up to $n-1$, i.e., assume that $\mathscr{P}(n-1)=\mathscr{C}^{2}(n-1)$ has been proved, where $n \geq 3$. It suffices to prove that $x^{\alpha}=x_{1}^{\alpha_{1}} \cdots x_{n}^{\alpha_{n}} \in \mathscr{H}^{2}(n)$ for all multi-indices $\alpha=\left(\alpha_{1}, \ldots, \alpha_{n}\right)$ and since, 
by the induction hypothesis, $x_{1}^{\alpha_{1}} \cdots x_{n-1}^{\alpha_{n-1}} \in \mathscr{H}^{2}(n-1)$ all reduces: Let $P$, $Q \in \mathscr{H}(n-1) ;$ then

$$
P\left(x_{1}, \ldots, x_{n-1}\right) Q\left(x_{1}, \ldots, x_{n-1}\right) x_{n}^{k} \in \mathscr{H}^{2}(n)
$$

for all integers $k \geq 0$. We proceed by induction on $k$. That (7) is valid is trivial if $k=0,1$ or 2 , so assume $k>2$ and (7) proved up to $k-1$. Let $m=[k / 2]$ and define inductively polynomials $T_{i} \in \mathscr{P}(n-1)$ by $\Delta T_{1}=k(k-1) P$ and $\Delta T_{j}=-(k-2 j+2)(k-2 j+1) T_{j-1}$ if $1<j \leq m$. Setting

$$
R=P x_{n}^{k}-\sum_{j=1}^{m} T_{j} x_{n}^{k-2 j},
$$

we see that $\Delta R=0$. Thus

$$
P Q x_{n}^{k}=R Q+\sum_{j=1}^{m} T_{j} Q x_{n}^{k-2 j} .
$$

All terms in this expression for $P Q x_{n}^{k}$ are in $\mathscr{H}^{2}(n)$, the first one because $R$, $Q$ are harmonic and the remaining ones because they only involve powers of $x_{n}$ of order $<k$. This concludes the proof of the theorem.

\section{REFERENCES}

1. A. G. Ramm, On completeness of the products of harmonic functions, Proc. Amer. Math. Soc. 98 (1986), 253-256.

2. $\ldots$, Recovery of the potential from fixed-energy scattering data, Inverse Problems 4 (1988), 877-886; 5 (1989), 255.

3. to PDE, J. Math. Anal. Appl. 134 (1988), 211-253; 136 (1988) 568-574; 139 (1989), 302.

4. __ Multidimensional inverse scattering problems and completeness of the products of solutions to homogeneous PDE, Zeitschr. Angew. Math. Mech. 69 (1969), T13-T22.

5. _ A simple proof of uniqueness theorem in impedance tomography, Appl. Math. Lett. 1 (1988), 287-290.

6. W. Rudin, Real and complex analysis, 3rd ed., McGraw-Hill, New York, 1987.

7. E. M. Stein, Singular integrals and differentiability properties of functions, Princeton University Press, Princeton, 1970.

Department of Mathematics, Florida Atlantic University, Boca Raton, Florida 33431 